\title{
Differential diagnostic value of computed tomography perfusion combined with vascular endothelial growth factor expression in head and neck lesions
}

\author{
JIE WANG $^{1 *}$, ZUOHUA TANG $^{1 *}$, SHUYI WANG ${ }^{2}$, WENJIAO ZENG $^{3}$, WEN QIAN $^{1}$, \\ LINGJIE WU ${ }^{1}$, WENZHONG WANG ${ }^{1}$ and JIANFENG LUO ${ }^{4}$
}

\begin{abstract}
Departments of ${ }^{1}$ Radiology, and ${ }^{2}$ Pathology, Eye Ear Nose and Throat Hospital, Fudan University, Shanghai 200031;
${ }^{3}$ Department of Pathology, Shanghai Medical School of Fudan University; ${ }^{4}$ Department of Health Statistics and Social Medicine, School of Public Health, Fudan University, Shanghai 200032, P.R. China
\end{abstract}

Received January 21, 2015; Accepted March 8, 2016

DOI: $10.3892 / \mathrm{ol} .2016 .4413$

\begin{abstract}
There are numerous types of head and neck lesions (HNLs), and conventional computed tomography (CT) has low specificity and sensitivity in the definitive and differential diagnosis of HNLs. The aim of the present study was to evaluate the value of perfusion CT (CTP) combined with vascular endothelial growth factor (VEGF) expression in the differentiation between malignant and benign HNLs. In total, 41 HNLs, which were pathologically confirmed, underwent CTP and VEGF expression analysis. All lesions were divided into three groups: Group A, benign hypovascular lesions; Group B, benign hypervascular lesions; and Group C, malignant lesions. Time density curve (TDC) and CTP parameters [maximum intensity projection (MIP), blood volume (BV), blood flow (BF), mean transit time and capillary permeability] were analyzed. The association between perfusion measurements and VEGF was assessed using Pearson's correlation. TDCs were classified into three types, and type I was more frequently identified in benign tumors (Groups A and B) compared with malignant tumors (Group C) $(\mathrm{P}=0.003)$. Malignant tumors primarily had a TDC of type II and III. MIP, BF and BV were all significantly higher in Groups B and $\mathrm{C}$ compared to Group $\mathrm{A}(\mathrm{P}<0.01)$. VEGF
\end{abstract}

Correspondence to: Dr Zuohua Tang, Department of Radiology, Eye Ear Nose \& Throat Hospital, Fudan University, 83 Fenyang Road, Shanghai 200031, P.R. China

E-mail: tzh518sunny@163.com

Dr Shuyi Wang, Department of Pathology, Eye Ear Nose and Throat Hospital, Fudan University, 83 Fenyang Road, Shanghai 200031, P.R. China

E-mail: wangshuyi@eent.shmu.edu.cn

*Contributed equally

Key words: head and neck lesions, angiogenesis, perfusion, computed tomography, vascular endothelial growth factor expression of malignant tumors was significantly higher than benign tumors $(\mathrm{P}=0.007)$. No correlation was identified between VEGF and any CTP parameter. The present findings suggest that CTP combined with VEGF may differentiate between malignant and benign HNLs, and between benign hypovascular and hypervascular lesions.

\section{Introduction}

Tumor angiogenesis is the process of novel blood vessel formation from existing vessels, which is critical for tumor growth, progression and metastasis (1). Several studies have revealed that tumor angiogenesis has prognostic value; there is an association between angiogenesis and increased risk of local regional recurrence, distant metastasis and decreased survival in patients with head and neck cancer (2-4). Traditionally, vascular endothelial cell growth factor (VEGF) staining of tumor tissues has been used to evaluate angiogenesis $(5,6)$. It has been reported that VEGF is a prognostic factor for the survival of patients with esophageal squamous cell carcinoma, and positive expression of VEGF is associated with a poorer prognosis $(5,7,8)$.

Current advances in functional imaging techniques have resulted in perfusion computed tomography (CTP), which is an attractive technique to assess tumor vascularity and indirectly reflects tumor angiogenesis (9-11). The development of novel vessels leads to physiological changes, and tumor angiogenesis is associated with increased perfusion, blood volume and permeability, which alters contrast enhancement during CTP. CTP quantifies these physiological changes and identifies the extent of tumor angiogenesis in the head and neck (12). In theory, the degree of neovascularity is different between benign and aggressive lesions. Consequently, CTP aids in the early detection of malignancy, assessment of responses to therapy and differentiation between residual or recurrent tumors and fibrous post-therapeutic alterations (11,13-15). As a clinical tool, CTP has been a promising technique in evaluating the angiogenesis of head and neck lesions (HNLs) in vivo and in differentiating malignant from benign lesions $(5,13,16,17)$. 
VEGF is an angiogenic molecular marker $(7,8)$, and CTP provides a quantitative measure of tumor perfusion, which reflects tumor angiogenesis (9-11). Therefore, CTP findings may be a predictive indicator of intratumoral VEGF. There have been a few studies evaluating the use of CTP in the differential diagnosis between benign and malignant HNLs $(13,16,17)$. However, to the best of our knowledge, there has been only one study concerning the correlation of CTP with VEGF in a group of HNLs, which demonstrated a positive correlation between MTT and VEGF (17). The aim of the present study was to prospectively study the correlation of CTP parameters with VEGF expression, and determine the value of CTP combined with VEGF in the differential diagnosis between malignant and benign HNLs.

\section{Materials and methods}

Patients. The current prospective study was performed with approval from the Institutional Review Board affiliated to the Eye Ear Nose \& Throat Hospital, Fudan University (Shanghai, China), and informed written consent was obtained from each patient prior to enrolment. In total, 41 patients $(23$ men and 18 women; age range, 25-74 years; mean age \pm standard deviation, 45.22 \pm 13.82 years) with HNLs were included in this study, who underwent surgical resection for HNLs from October 2008 and March 2010. Primary tumor sites consisted of the parotid gland $(n=20)$, temporal bone $[n=11$, including external auditory canal $(n=7)$, mastoid process $(n=3)$ and petrous portion $(n=1)]$, parapharyngeal space $(n=7)$, infratemporal fossa $(n=1)$, jugular foramen $(n=1)$ and pterygopalatine fossa $(n=1)$. All the histopathological diagnoses were confirmed by surgical specimens.

CTP imaging. CT scans were performed with a 16-row multi-slice CT scanner (Somatom 16, Sensation; Siemens Healthcare, Erlangen, Germany). An unenhanced CT of the neck was performed prior to CTP. On transverse CT images, the slice revealing the maximal transverse tumor diameter was selected to measure perfusion. Two adjacent $12-\mathrm{mm}$ sections were selected at the level of the tumor. A total of $50 \mathrm{ml}$ nonionic iodinated contrast agent $(300 \mathrm{mg} / \mathrm{ml}$; Ultravist 300 ; Bayer Health Care, Whippany, NJ, USA) was injected at $4 \mathrm{ml} / \mathrm{sec}$ using a Optivantage power injector (Cincinnati, $\mathrm{OH}$, USA). At $4 \mathrm{sec}$ into the injection, a continuous acquisition was initiated using the following parameters: $80 \mathrm{kV}, 100 \mathrm{~mA}$, $2 \times 12 \mathrm{~mm}$ sections, with a temporal resolution of 1 picture $/ \mathrm{sec}$ for a $40 \mathrm{sec}$ duration. Following the completion of the perfusion acquisition, dual-phase imaging (arterial and venous acquisitions) was performed. Fixed delays of 25-30 sec for the arterial phase and $90 \mathrm{sec}$ for the venous phase were included. To increase radiation protection, the volume acquisition was reduced to the area of the lesion in the arterial phase. The venous phase scanning (routine scanning) was between the skull base and thoracic inlet. All the lesions were subdivided into benign (Group A and B) and malignant lesions (Group C) upon pathological examination (Table I). Subsequently, two head and neck radiologists (Department of Radiology, Eye Ear Nose \& Throat Hospital, Shanghai, China; 25 years of experience in radiology) blindly reviewed the dual-phase images and divided the benign lesions into two groups (Group A, benign hypovascular lesions; Group B, benign hypervascular lesions) (Table I). Imaging criteria for benign hypervascular lesions consisted of arterial contrast enhancement and washout on delayed images. For benign hypovascular lesions imaging features were defined as delayed enhancement of the venous phase, which had no obvious enhancement on the arterial phase (18).

CTP imaging postprocessing. Quantitative evaluation of the CTP data was performed in consensus by the two radiologists, using commercially available body perfusion software (syngo ${ }^{\circledR}$ Volume Perfusion CT Body; Siemens Healthcare). The Patlak approach was used for analysis (19). After the CT data set was corrected for motion with an integrated motion correction algorithm (20), a circular region of interest (ROI) was placed in the input artery, either the ipsilateral external or internal carotid artery, since there was substantial anatomic variation, which rendered it challenging to use the same artery in different patients. Subsequently, color coded maps of maximum intensity projection (MIP), blood volume (BV), blood flow (BF), mean transit time (MTT) and capillary permeability $(\mathrm{CP})$ were generated. A ROI was drawn within the greatest dimension plane of the tumor (range, $19-1032 \mathrm{~mm}^{2}$ ). If homogeneous enhancement was identified, the ROI was drawn to cover $\geq 50 \%$ of the tumor surface. If heterogeneous enhancement was identified, the regions with the highest enhancement were selected. Care was taken to avoid regions of adjacent normal vasculature and those that had no enhancement, including calcification and cystic, necrotic and hemorrhagic components (21). Subsequently, the time density curves (TDCs) and the five parametric values of CTP (MIP, BV, BF, MTT and CP) were generated for selected ROIs. For CTP imaging post-processing, the commercially available body perfusion software (Syngo Volume Perfusion CT Body, VA 40; Siemens Healthcare) was used.

VEGF immunohistochemistry and assessment. All patients underwent surgical resection as soon as possible following the CTP scan. Two pathologists and two radiologists jointly performed the processing of all surgical specimens and matching with CT images. Subsequent to a $24 \mathrm{~h}$ fixation in formalin (Sinopharm Chemical Reagent Co., Ltd, Shanghai, China), the surgical specimens were sliced in the transverse plane at the level of the maximal tumor diameter (range, 0.9-5.7 cm). The macroscopic appearance of the transversely sliced surgical specimen was compared with the appearance of the corresponding tumor plane on transverse $\mathrm{CT}$ images to ensure maximal consistency of the regions subjected to pathological examination with the ROIs selected on the CT images. Subsequently a 3-5 mm thick slice was cut from the specimen in this plane, and all tissue slices were embedded in paraffin. From the paraffin, $4-\mu \mathrm{m}$ thick sections were cut and incubated with phosphate-buffered saline (Sinopharm Chemical Reagent Co., Ltd), 3\% $\mathrm{H}_{2} \mathrm{O}_{2}$ solution (Sinopharm Chemical Reagent Co., Ltd) and citrate buffer (Sinopharm Chemical Reagent Co., Ltd). The Histostain ${ }^{\mathrm{TM}}$ Plus Kit (Fuzhou Maixin Biological Technology Development Co., Ltd., Fuzhou, China), rabbit anti-VEGF polyclonal antibody (dilution, 1:100; catalog no. ab46154; Abcam, Cambridge, MA, USA) and DAB Horseradish Peroxidase Color Development Kit (Fuzhou Maixin 
Table I. Histopathological diagnosis, TDC types and CTP parameters in 41 patients with head and neck lesions.

\begin{tabular}{|c|c|c|c|c|c|c|c|c|c|}
\hline \multirow[b]{2}{*}{ Histopathologic diagnosis } & \multirow[b]{2}{*}{ Total, n } & \multicolumn{3}{|c|}{ TDC types, $\mathrm{n}$} & \multicolumn{5}{|c|}{ CTP parameters } \\
\hline & & $\mathrm{I}$ & II & III & $\begin{array}{l}\mathrm{MIP}, \\
\mathrm{Hu}\end{array}$ & $\begin{array}{c}\mathrm{BV}, \\
\mathrm{ml} / 100 \mathrm{ml}\end{array}$ & $\begin{array}{c}\mathrm{BF}, \\
\mathrm{ml} / 100 \mathrm{ml} / \mathrm{min}\end{array}$ & $\begin{array}{l}\text { MTT, } \\
\text { sec } \quad \text { r }\end{array}$ & $\begin{array}{c}\mathrm{CP} \\
\mathrm{ml} / 100 \mathrm{ml} / \mathrm{min}\end{array}$ \\
\hline Benign lesions $\left(\mathrm{G}_{\mathrm{A}+\mathrm{B}}\right)$ & 29 & 14 & 4 & 11 & $73.8 \pm 34.1$ & $6.7 \pm 7.2$ & $73.7 \pm 51.1$ & $9.4 \pm 4.1$ & $1 \quad 26.6 \pm 23.1$ \\
\hline Benign hypovascular lesions $\left(\mathrm{G}_{\mathrm{A}}\right)$ & 16 & 12 & 0 & 4 & $55.3 \pm 30.1$ & $2.8 \pm 2.2$ & $39.1 \pm 19.9$ & $9.9 \pm 4.2$ & $17.6 \pm 11.3$ \\
\hline Pleomorphic adenoma & 9 & 6 & 0 & 3 & $59.0 \pm 12.6$ & $3.1 \pm 2.5$ & $42.5 \pm 23.7$ & $8.6 \pm 3.3$ & $20.6 \pm 8.9$ \\
\hline Lymphoepithelial cyst & 2 & 2 & 0 & 0 & 38.8 & 2.1 & 32.7 & 9.1 & 3.9 \\
\hline Branchial cleft cyst & 2 & 2 & 0 & 0 & 25.6 & 2.7 & 38.6 & 11.9 & 24.7 \\
\hline Schwannoma & 1 & 1 & 0 & 0 & 28.5 & 1.0 & 38.0 & 8.0 & 3.7 \\
\hline Venous hemangioma & 1 & 1 & 0 & 0 & 48.5 & 0.6 & 19.5 & 13.6 & 4.0 \\
\hline Osteochondroma & 1 & 0 & 0 & 1 & 147.8 & 5.6 & 43.6 & 17.8 & 31.2 \\
\hline Benign hypervascular lesions $\left(G_{B}\right)$ & 13 & 2 & 4 & 7 & $96.5 \pm 23.6$ & $11.5 \pm 8.4$ & $116.2 \pm 45.1$ & $8.7 \pm 4.1$ & $1 \quad 37.7 \pm 28.9$ \\
\hline Kimura's disease & 3 & 0 & 0 & 3 & $79.3 \pm 14$ & $9.4 \pm 5.2$ & $101.5 \pm 45.8$ & $8 \pm 3.9$ & $35.3 \pm 0.9$ \\
\hline Warthin's tumor & 2 & 0 & 2 & 0 & 108.7 & 23.1 & 174.9 & 5.3 & 20.6 \\
\hline Papillary epithelioma & 2 & 0 & 2 & 0 & 97.8 & 7.9 & 97.1 & 7.4 & 25.5 \\
\hline Intradermal nevus & 1 & 0 & 0 & 1 & 75.9 & 22.1 & 164.8 & 17.7 & 119.7 \\
\hline Chondroblastoma & 1 & 0 & 0 & 1 & 142.1 & 6.8 & 96.2 & 6.3 & 39.9 \\
\hline Glomus tumor & 1 & 1 & 0 & 0 & 78.4 & 4.5 & 73.7 & 7.4 & 45.7 \\
\hline Granulosa cell tumor & 1 & 0 & 0 & 1 & 77.9 & 3.8 & 44.4 & 13.3 & 22.8 \\
\hline Basal cell adenoma & 1 & 0 & 0 & 1 & 134.2 & 14.2 & 156.6 & 12.9 & 0.4 \\
\hline Giant cell granuloma & 1 & 1 & 0 & 0 & 95.4 & 8.0 & 127.2 & 6.7 & 64.4 \\
\hline Malignant lesions $\left(\mathrm{G}_{\mathrm{C}}\right)$ & 12 & 0 & 6 & 6 & $79.1 \pm 25.1$ & $12.9 \pm 12.5$ & $112.4 \pm 76.6$ & $8.4 \pm 2.8$ & $8 \quad 33.2 \pm 22.6$ \\
\hline Rhabdomyosarcoma & 2 & 0 & 2 & 0 & 77.9 & 18.7 & 185.2 & 6.6 & 49.7 \\
\hline Adenoid cystic carcinoma & 2 & 0 & 0 & 2 & 76.5 & 4.8 & 48.9 & 10.0 & 21.9 \\
\hline Malignant schwannoma & 2 & 0 & 1 & 1 & 65.3 & 10.7 & 112.1 & 6.7 & 40.5 \\
\hline Mucoepidermoid carcinoma & 1 & 0 & 1 & 0 & 145.7 & 45.9 & 241.2 & 10.1 & 21.8 \\
\hline Squamous cell carcinoma & 2 & 0 & 2 & 0 & 88.1 & 13.7 & 120.6 & 12.1 & 41.5 \\
\hline WDC & 1 & 0 & 0 & 1 & 43.4 & 1.4 & 37.2 & 4.3 & 15.4 \\
\hline Epithelioid sarcoma & 1 & 0 & 0 & 1 & 79.5 & 7.1 & 57.5 & 9.3 & 17.8 \\
\hline MEMT & 1 & 0 & 0 & 1 & 65.6 & 5.0 & 79.3 & 6.6 & 36.7 \\
\hline
\end{tabular}

Data are presented as the mean \pm standard deviation. TDC, time density curve; CTP, computed tomography perfusion; MIP, maximum intensity projection; BV, blood volume; BF, blood flow; MTT, mean transit time; $\mathrm{CP}$, capillary permeability; $\mathrm{G}_{\mathrm{A}+\mathrm{B}}, \mathrm{Groups}_{\mathrm{A}}$ and $\mathrm{B} ; \mathrm{G}_{\mathrm{A}}$, Group A; $\mathrm{G}_{\mathrm{B}}$, Group B; $\mathrm{G}_{\mathrm{C}}$, Group C; WDC, well-differentiated chondrosarcoma; MEMT, metastatic epithelial malignant tumor.

Biological Technology Development Co., Ltd.) were used for the detection of VEGF. VEGF expression was visualized using a microscope (Eclipse Ci; Nikon Corporation, Tokyo, Japan). In addition to histopathological analysis, routine tissue samples for hematoxylin and eosin staining (Shanghai Hongqiao Le Xiang Medical Technology Co., Ltd, Shanghai, China) were also obtained. All the immunohistochemical results were evaluated by the two pathologists, and consensus was reached when there was a discrepancy.

Assessment of VEGF. According to the criteria established by Volm et al (22) for evaluating VEGF expression, a score was designated corresponding to the sum of the following: (a) Percentage of positive cells $(0,0 \%$ immunopositive cells; $1,<25 \%$ positive cells; $2,26-50 \%$ positive cells; and 3 , $>50 \%$ positive cells); and (b) Staining intensity ( 0 , negative; 1, weak; 2 , moderate; and 3 , high). The sum of $a+b$ had $a$ maximum score of 6 . Scores between 0 and 2 were regarded as negative, scores of 3 and 4 as weakly positive and scores between 5 and 6 as strongly positive. At low magnification (x40), 10 fields with the highest density of VEGF positive expression in the tumor cells were initially determined. The 10 fields were composed of 6 counting regions in the central portion and 4 in the peripheral portion; necrotic areas were avoided. VEGF staining extent and intensity were assessed in each of 10 fields at high magnification (x400) for each field. The total scores for 10 fields were averaged and recorded as the final score of VEGF expression for each tumor specimen.

Statistical analysis. SPSS version 16.0 (SPSS, Inc., Chicago, IL, USA) was used for all statistical analysis. $\mathrm{P}<0.05$ was considered to indicate a statistically significant difference. The Mann-Whitney test was used in the comparison of CT 

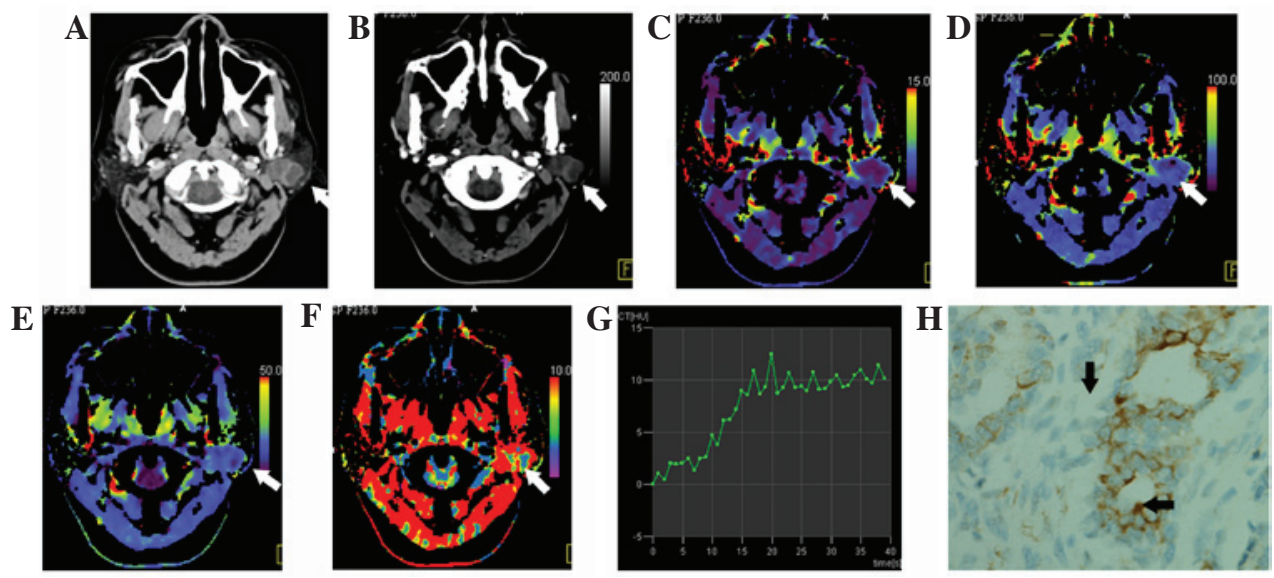

Figure 1. A 50-year-old female of Group A with pleomorphic adenoma in the left parotid. (A) Axial contrast-enhanced CT shows an irregular mass with heterogeneous enhancement (arrow). (B-F) CT perfusion functional maps show that the lesion has relatively (B) increased MIP, and decreased (C) BV, (D) BF, (E) CP and (F) MTT compared with the contralateral normal parotid gland. (G) Time density curve of type I; a steep ascending phase with no descending phase. (H) Vascular endothelial growth factor expression is weakly positive; brown staining is observed in the tumour cytoplasm and cytomembrane (arrow; magnification, x400). CT, computed tomography; MIP, maximum intensity projection; BV, blood volume; BF, blood flow; MTT, mean transit time; CP, capillary permeability.
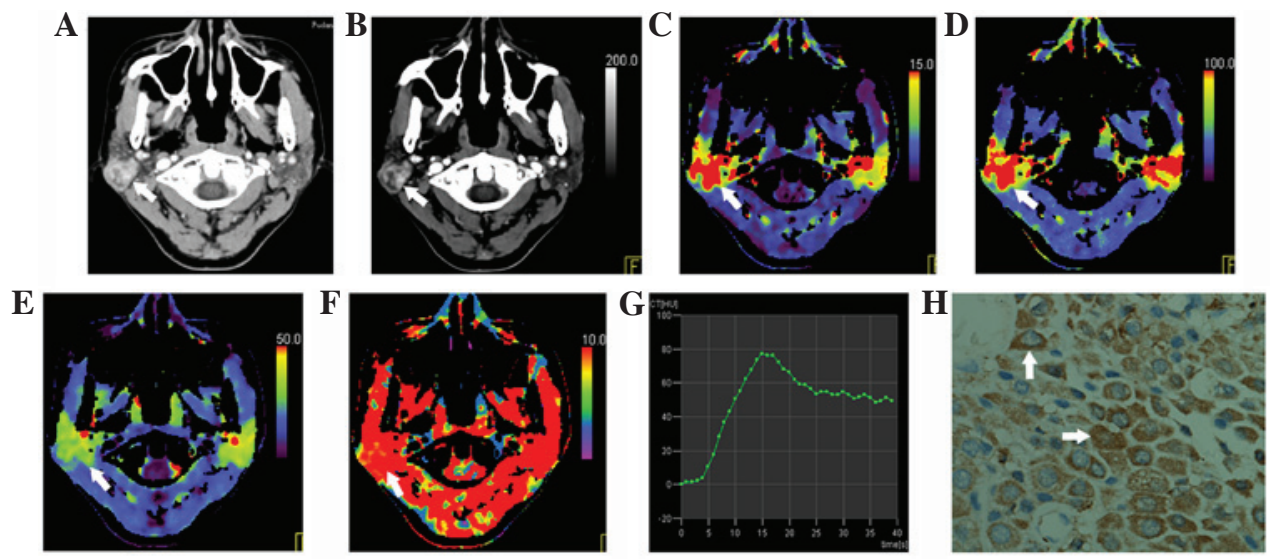

Figure 2. A 50-year-old female of Group C with a mucoepidermoid carcinoma in the right parotid. (A) Axial contrast-enhanced CT shows an ill-defined mass with heterogeneous enhancement (arrow). (B-F) CT perfusion functional maps show that the lesion has relatively increased (B) MIP, (C) BV and (D) BF, and decreased (E) CP and (F) MTT compared with the contralateral normal parotid gland. (G) Time density curve of type II with a steep ascending phase and an apparent descending phase. (H) VEGF expression is strongly positive; brown staining was observed in the tumour cytoplasm (arrow; magnification, $\mathrm{x} 400$ ). CT, computed tomography; MIP, maximum intensity projection; BV, blood volume; BF, blood flow; MTT, mean transit time; $\mathrm{CP}$, capillary permeability.

perfusion parameters and VEGF expression between any two groups. Pearson's correlation coefficients were calculated to assess the correlation between perfusion measurements (MIP, $\mathrm{BV}, \mathrm{BF}, \mathrm{MTT}, \mathrm{CP}$ ) and VEGF.

\section{Results}

Characteristics of dynamic enhancement TDC. The obtained TDCs were classified into three types on the basis of the time to peak (TTP) and the washout ratio (WR) (23). These TDC parameters were calculated using the following equations: TTP = time required to reach the enhancement density peak; WR $(\%)=[($ density at peak)-(density at $44 \mathrm{sec}$ after the start of contrast medium injection)] x $100 /$ [(density at peak)-(density at the start of contrast medium injection)] (21). The three types of TDC were classified as follows: Type I TDCs, TTP $>30 \mathrm{sec}$ and no washout; type II, TTP $\leq 30 \mathrm{sec}$ and $\mathrm{WR}>30 \%$; type III,
TTP $<30$ sec and WR $<30 \%$. Table I summarizes the various types of TDC in the three groups.

TDC of type I (Fig. 1) was more frequently identified in Group A compared with Groups B and C $(\mathrm{P}=0.003$ and $\mathrm{P}<0.001)$ respectively). Type II (Fig. 2) was only identified in Groups B and C. There was no significant difference in TDC of type III among the three groups. TDC of type I was more frequently identified in benign tumors (Groups A and B) compared with malignant tumors (Group C) $(\mathrm{P}=0.003)$. Malignant tumors primarily had TDC of type II and III.

CTP parameters. The values of the CTP parameters for each tumor are presented in Table I, and the differences between the CTP parameters among the three groups are summarized in Table II. MIP, BV and BF were all statistically increased in Groups B and C compared with Group A (Table II). CP was increased in Group B compared with Group A $(\mathrm{P}=0.002)$. Group B had increased MIP values compared with Group C $(\mathrm{P}=0.044)$. 
Table II. Comparison of computed tomography perfusion parameters and VEGF among the three groups of head and neck patients.

\begin{tabular}{|c|c|c|c|c|c|c|}
\hline Group & $\begin{array}{c}\mathrm{MIP}, \\
\mathrm{Hu}\end{array}$ & $\begin{array}{c}\mathrm{BV}, \\
\mathrm{ml} / 100 \mathrm{ml}\end{array}$ & $\begin{array}{c}\mathrm{BF} \\
\mathrm{ml} / 100 \mathrm{ml} / \mathrm{min}\end{array}$ & $\begin{array}{l}\text { MTT, } \\
\text { sec }\end{array}$ & $\begin{array}{c}\mathrm{CP} \\
\mathrm{ml} / 100 \mathrm{ml} / \mathrm{min}\end{array}$ & $\begin{array}{c}\text { VEGF } \\
\text { expression }\end{array}$ \\
\hline \multicolumn{7}{|l|}{ A } \\
\hline Range & $21.6-147.8$ & $0.5-9.2$ & $15.8-83.6$ & $3.0-17.8$ & $1.2-33.8$ & $0-5.5 .0$ \\
\hline $\mathrm{M}$ & 55.3 & 2.3 & 35.8 & 9.8 & 16.0 & 3.4 \\
\hline \multicolumn{7}{|l|}{ B } \\
\hline Range & $64.1-142.1$ & $3.8-33.5$ & 44.4-188.6 & $3.0-17.7$ & $0.4-119.7$ & $1.0-4.8$ \\
\hline M & 91.7 & 8.0 & 105.2 & 7.6 & 34.5 & 3.7 \\
\hline \multicolumn{7}{|l|}{$\mathrm{C}$} \\
\hline Range & $43.4-145.7$ & $1.4-45.9$ & $37.2-249.4$ & $4.3-13.5$ & $15.2-81.5$ & $3.0-5.6$ \\
\hline M & 76.4 & 9.1 & 87.2 & 9.2 & 23.9 & 5.0 \\
\hline \multicolumn{7}{|l|}{ A vs. B } \\
\hline Z score & -3.667 & -3.946 & -3.992 & -1.323 & -3.064 & -0.256 \\
\hline P-value & $<0.001^{\mathrm{a}}$ & $<0.001^{\mathrm{a}}$ & $<0.001^{\mathrm{a}}$ & 0.186 & $0.002^{\mathrm{a}}$ & 0.798 \\
\hline \multicolumn{7}{|l|}{ A vs. C } \\
\hline Z score & -2.693 & -3.389 & -3.296 & -0.882 & -1.811 & -2.262 \\
\hline P-value & $0.007^{\mathrm{a}}$ & $0.001^{\mathrm{a}}$ & $0.001^{\mathrm{a}}$ & 0.378 & 0.070 & $0.024^{\mathrm{b}}$ \\
\hline \multicolumn{7}{|l|}{ B vs. C } \\
\hline Z score & -2.013 & -0.272 & -0.761 & -0.082 & -0.870 & -2.510 \\
\hline P-value & $0.044^{\mathrm{b}}$ & 0.786 & 0.446 & 0.935 & 0.384 & $0.012^{\mathrm{b}}$ \\
\hline \multicolumn{7}{|l|}{$\mathrm{A}+\mathrm{B}$ vs. $\mathrm{C}$} \\
\hline Z score & -0.602 & -1.948 & -1.633 & -0.501 & -0.659 & -2.713 \\
\hline P-value & 0.547 & 0.051 & 0.102 & 0.616 & 0.510 & $0.007^{\mathrm{a}}$ \\
\hline
\end{tabular}

Mann Whitney $\mathrm{U}$ test used for statistical analysis. ${ }^{\mathrm{a}} \mathrm{P}<0.01,{ }^{\mathrm{b}} \mathrm{P}<0.05$. A, benign hypovascular lesions; $\mathrm{B}$, benign hypervascular lesions; $\mathrm{C}$, malignant lesions; M, median; MIP, maximum intensity projection; BV, blood volume; BF, blood flow; MTT, mean transit time; CP, capillary permeability; VEGF, vascular endothelial growth factor.

Table III. Pearson's correlation for computed tomography perfusion parameters and VEGF.

\begin{tabular}{|c|c|c|c|c|c|c|c|c|c|c|}
\hline \multirow{2}{*}{$\begin{array}{l}\text { VEGF } \\
\text { expression }\end{array}$} & \multicolumn{2}{|c|}{$\begin{array}{c}\mathrm{MIP}, \\
\mathrm{Hu}\end{array}$} & \multicolumn{2}{|c|}{$\begin{array}{c}\mathrm{BV}, \\
\mathrm{ml} / 100 \mathrm{ml}\end{array}$} & \multicolumn{2}{|c|}{$\begin{array}{c}\mathrm{BF} \\
\mathrm{ml} / 100 \mathrm{ml} / \mathrm{min}\end{array}$} & \multicolumn{2}{|c|}{$\begin{array}{l}\text { MTT, } \\
\text { sec }\end{array}$} & \multicolumn{2}{|c|}{$\begin{array}{c}\mathrm{CP} \\
\mathrm{ml} / 100 \mathrm{ml} / \mathrm{min}\end{array}$} \\
\hline & $\mathrm{r}$ & $\mathrm{P}$ & $\mathrm{r}$ & $\mathrm{P}$ & $\mathrm{r}$ & $\mathrm{P}$ & $\mathrm{r}$ & $\mathrm{P}$ & $\mathrm{r}$ & $\mathrm{P}$ \\
\hline++ & 0.292 & 0.312 & 0.283 & 0.327 & 0.408 & 0.148 & -0.466 & 0.100 & 0.100 & 0.734 \\
\hline+ & 0.106 & 0.640 & 0.048 & 0.830 & 0.019 & 0.933 & 0.108 & 0.600 & 0.170 & 0.448 \\
\hline- & -0.498 & 0.393 & -0.002 & 0.998 & 0.004 & 0.996 & 0.349 & 0.600 & -0.129 & 0.837 \\
\hline
\end{tabular}

++, strong positive; +, weakly positive; -, negative; P, P-value; r, correlation coefficient; MIP, maximum intensity projection; BV, blood volume; BF, blood flow; MTT, mean transit time; CP, capillary permeability; VEGF, vascular endothelial growth factor.

VEGF expression and its association with CTP parameters. The results of VEGF expression in the three groups are presented in Table II. The Mann-Whitney test revealed a significant difference of VEGF expression in Group $\mathrm{C}$ vs. A $(\mathrm{P}=0.024)$ and Group $\mathrm{C}$ vs. $\mathrm{B}(\mathrm{P}=0.012)$. VEGF expression of malignant tumors (Group $C$ ) was significantly higher compared with benign tumors (Groups A $+\mathrm{B}$; $\mathrm{P}=0.007$ ). The correlation between perfusion parameters and VEGF is presented in Table III. VEGF expression was not correlated with any CTP parameters $(\mathrm{P}>0.05)$.

\section{Discussion}

CTP is performed to evaluate blood flow patterns of lesions, and the various appearances of the TDCs of HNLs primarily represent the differences in blood perfusion (17). In the present study, TDC of type I was more frequently identified in Group A (benign hypovascular lesions) compared with Group B (benign hypervascular lesions) $(\mathrm{P}=0.003)$, which indicated that TDC of type I may be used to distinguish between benign hypovascular and hypervascular lesions. In addition, TDC of type I 
was more frequently identified in benign tumors (Groups A and $\mathrm{B}$ ) compared with malignant tumors (Group $\mathrm{C})(\mathrm{P}=0.003)$. This may be due to the decreased blood supply and intact vascular wall observed benign tumors, particularly in benign hypovascular lesions (17). Consequently, TDC of type I may aid in the differentiation between benign and malignant HNLs. Additionally in the present study, malignant tumors primarily were TDC type II and III, and type II was only identified in Groups B and C. In malignant tumors in the present study, due to the abundant novel vessels, loosely interconnected endothelium and immature basement membrane (17), the contrast agent dispersed quickly following injection. Therefore, these were classified as TDC of type II, and may be used to differentiate Group A from Groups B and C. In addition, certain tumors in Group B, including Warthin's tumors and papillary epitheliomas, also had TDC of type II, which may be attributed pathologically to their extensive capillary networks and numerous leaky blood vessels (24). Therefore, the various types of TDC may reflect the alterations in blood microcirculation and may aid in distinguishing between benign and malignant HNLs, and between benign hypovascular and hypervascular lesions.

There are three main differences in CTP parameters in the differentiation of malignant and benign HNLs $(13,16,17)$. Rumboldt et al (16) demonstrated that benign head and neck lesions had a lower BF and longer MTT compared with malignant lesions. Conversely, Bisdas et al (13) reported that the BV and $\mathrm{BF}$ of benign parotid tumors were significantly higher than those of malignant tumors. In the present study, MIP, BV and $\mathrm{BF}$ were all statistically higher in Groups B and C compared with Group A $(\mathrm{P}<0.01)$. As MIP may reflect the overall level and average degree of perfusion, its increase in Groups B and $\mathrm{C}$ may reveal more abundant angiogenesis than Group A. A significantly high $\mathrm{BV}$ in Groups $\mathrm{B}$ and $\mathrm{C}$ may reflect an increased vascular bed in the process of angiogenesis (the growth of novel vessels from pre-existing ones), while a high $\mathrm{BF}$ may reflect the formation of a number of arteriovenous shunts in tumor angiogenesis. Therefore, MIP, BV and BF reflect the extent of tumor angiogenesis in HNLs and may be of great value in the differentiation of Group A from Groups B and $\mathrm{C}$. However, there were no significant differences in $\mathrm{BV}$ and $\mathrm{BF}$ between Group B and Group C ( $>>0.05)$. This may be due to the numerous microvessels and high cellularity-stromal grade observed in benign hypervascular lesions, which results in a high WR (13), followed by MIP, BV and BF increases. Additionally in the present study, the necrotic regions of malignant tumors had an apparent affect on CTP parameters, which could reduce MIP, BV and BF. Furthermore, CP was higher in Group B than in Group A $(\mathrm{P}=0.002)$, and this may aid in differentiating between benign hypovascular and hypervascular lesions. The difference may be due to the leaky blood vessels of certain tumors in Group B, including Warthin's tumors and papillary epitheliomas (24), which has led to the capillary permeability increase.

The importance of angiogenesis in tumor growth is well-established (14). Although there are numerous types of vascular growth factors, VEGF is crucial in tumor angiogenesis due to its specialized role in promoting tumor karyokinesis (25). Yang et al (17) demonstrated that high VEGF expression was significantly different between malignant and benign HNLs.
In the present study, there were no significant differences in VEGF expression between Group A and Group B. However, the VEGF expression in Group $\mathrm{C}$ was significantly higher than that Groups $\mathrm{A}$ and $\mathrm{B}(\mathrm{P}=0.024$ and $\mathrm{P}=0.012$, respectively), which indicated that VEGF expression may be useful to differentiate between malignant and benign HNLs.

CTP assesses tumor vascularity quantitatively in vivo with vascular parameters, including BV, BF, MTT and CP, and it has been used as a tool for the assessment of antivascular chemotherapy $(15,26)$. Therefore, the vascular parameters of CTP may be an appropriate surrogate for angiogenesis and may have certain correlations with histological measures of angiogenesis, including VEGF.

Different results to the present study, have been demonstrated in prior studies that focused on the correlation between CTP parameters and VEGF expression. Zhang et al (27) revealed that there was a positive correlation between BV, BF, PS and VEGF, and a negative correlation between MTT and VEGF in a malignant rabbit VX2 liver tumor model. In addition, Yang et al (17) reported a significant correlation between VEGF expression and MTT in HNLs. In the present study, no correlation was observed between VEGF expression and any CTP measurements in all three groups, in accordance with the findings of previous studies where no association was demonstrated between the expression levels of VEGF and any perfusion CT parameter in colorectal cancer (28), gastric adenocarcinoma (29), and renal cell carcinoma (30). Although VEGF is a key stimulatory factor that promotes tumor angiogenesis (31), it is not the only factor that influences tumor angiogenesis. Other factors, including platelet-derived endothelial cell grow th factor, cyclooxygenase-2 and interleukin-8, also participate in tumor angiogenesis, which may lead to alterations in CTP parameters (31). Furthermore, VEGF expression is upregulated according to alterations in the microenvironment, including hypoxia and a decrease in $\mathrm{pH}$ (31), without an obvious impact on the results of tumor CTP. Consequently, it is possible that there is no correlation between VEGF expression and CTP measurements in HNLs; however, this requires validation.

The present study had certain limitations. First, due to the small sample size and overlap in the CTP data among the three groups, a larger scale trial is required to validate the differential diagnostic value of CTP in HNLs. Second, the perfusion scanning time $(40 \mathrm{sec})$ was not long enough in the present study, and prolonged scans may provide more information in the differential diagnosis of HNLs. Third, the CTP studies were performed on a 16-row multi-slice CT scanner, and more precise data would be acquired with thinner slices and a larger scanning area on a more advanced multidetector CT.

In conclusion, the present study suggests that CTP may differentiate between malignant and benign HNLs and between benign hypovascular and hypervascular HNLs. In addition, VEGF may differentiate between benign and malignant HNLs. Overall, CTP combined with VEGF may be beneficial for the differentiation, and reflect the angiogenesis, of HNLs.

\section{Acknowledgements}

The present study was supported by the Crossover Study Fund of Basic \& Clinical Medicine of Shanghai Medical School of Fudan University (Shanghai, China; grant no. Z-259). 


\section{References}

1. Lee TY, Purdie TG and Stewart E: CT imaging of angiogenesis. Q J Nucl Med 47: 171-187, 2003.

2. Li C, Fan J, Song X, Zhang B, Chen Y, Li C, Mi K, Ma H, Song Y, Tao $X$ and Li G: Expression of angiopoietin-2 and vascular endothelial growth factor receptor-3 correlates with lymphangiogenesis and angiogenesis and affects survival of oral squamous cell carcinoma. PLoS One 8: e75388, 2013.

3. Dai W, Li Y, Zhou Q, Xu Z, Sun C, Tan X and Lu L: Cetuximab inhibits oral squamous cell carcinoma invasion and metastasis via degradation of epidermal growth factor receptor. J Oral Pathol Med 43: 250-257, 2014

4. de Oliveira MV, Pereira Gomes EP, Pereira CS, de Souza LR, Barros LO, Mendes DC, Guimarães AL and De Paula AM: Prognostic value of microvessel density and p53 expression on the locoregional metastasis and survival of the patients with head and neck squamous cell carcinoma. Appl Immunohistochem Mol Morphol 21: 444-451, 2013.

5. Chen TW, Yang ZG, Chen HJ, Li Y, Tang SS, Yao J, Dong ZH and He D: Quantitative assessment of first-pass s using a low-dose method at multidetector CT in oesophageal squamous cell carcinoma: Correlation with VEGF expression. Clin Radiol 67: 746-753, 2012.

6. Ling S, Deng D, Mo Y, Zhang X, Guan X and Wei Q: Correlations between CT perfusion parameters and vascular endothelial growth factor expression and microvessel density in implanted VX2 lung tumors. Cell Biochem Biophys 70: 629-633, 2014.

7. Choi JY, Jang KT, Shim YM, Kim K, Ahn G, Lee KH, Choi Y, Choe YS and Kim BT: Prognostic significance of vascular endothelial growth factor expression and microvessel density in esophageal squamous cell carcinoma: Comparison with positron emission tomography. Ann Surg Oncol 13: 1054-1062, 2006.

8. Kleespies A, Guba M, Jauch KW and Bruns CJ: Vascular endothelial growth factor in esophageal cancer. J Surg Oncol 87: 95-104, 2004.

9. Meijerink MR, van Waesberghe JH, van der Weide L, van den Tol P, Meijer S and van Kuijk C: Total-liver-volume perfusion CT using 3-D image fusion to improve detection and characterization of liver metastases. Eur Radiol 18: 2345-2354, 2008.

10. Yi CA, Lee KS, Kim EA, Han J, Kim H, Kwon OJ, Jeong YJ and Kim S: Solitary pulmonary nodules: Dynamic enhanced multi-detector row CT study and comparison with vascular endothelial growth factor and microvessel density. Radiology 233 : 191-199, 2004.

11. Trojanowska A, Trojanowski P, Drop A, Jargiełło T and Klatka J: Head and neck cancer: Value of perfusion CT in depicting primary tumor spread. Med Sci Monit 18: CR112-CR118, 2012.

12. Miles KA: Tumour angiogenesis and its relation to contrast enhancement on computed tomography: A review. Eur J Radiol 30: 198-205, 1999.

13. Bisdas S, Baghi M, Wagenblast J, Knecht R, Thng CH, Koh TS and Vogl TJ: Differentiation of benign and malignant parotid tumours using deconvolution-based perfusion CT imaging: Feasibility of the method and initial results. Eur J Radiol 64: 258-265, 2007.

14. George ML, Dzik-Jurasz AS, Padhani AR, Brown G, Tait DM, Eccles SA and Swift RI: Non-invasive methods of assessing angiogenesis and their value in predicting response to treatment in colorectal cancer. Br J Surg 88: 1628-1636, 2001.

15. Sahani DV, Kalva SP, Hamberg LM, Hahn PF, Willett CG, Saini S, Mueller PR and Lee TY: Assessing tumour perfusion and treatment response in rectal cancer with multisection CT: Initial observations. Radiology 234: 785-792, 2005.

16. Rumboldt Z, Al-Okaili R and Deveikis JP: Perfusion $\mathrm{CT}$ for head and neck tumours: Pilot study. AJNR Am J Neuroradiol 26: 1178-1185, 2005.

17. Yang ZY, Meng QF, Xu QL, Li SR, Yan CG, Xie HB, Yang XF, Peng Q and Lai YR: Correlation between CT perfusion and vascular endothelial growth factor in neoplasm of head and neck. Zhonghua Fang She Xue Za Zhi 41: 900-906, 2007 (In Chinese).
18. Schmid-Tannwald C, Thomas S, Ivancevic MK, Dahi F, Rist C, Sethi I and Oto A: Diffusion-weighted MRI of metastatic liver lesions: Is there a difference between hypervascular and hypovascular metastases? Acta Radiol 55: 515-523, 2014

19. Spira D, Neumeister H, Spira SM, Hetzel J, Spengler W and von Weyhern $\mathrm{CH}$ : Assessment of tumor vascularity in lung cancer using volume perfusion CT (VPCT) with histopathologic comparison: A further step toward an individualized tumor characterization. J Comput Assist Tomogr 37: 15-21, 2013.

20. Reiner CS, Roessle M, Thiesler T, Eberli D, Klotz E and Frauenfelder T: Computed tomography perfusion imaging of renal cell carcinoma: Systematic comparison with histopathological angiogenic and prognostic markers. Invest Radiol 48: 183-191, 2013.

21. d'Assignies G, Couvelard A, Bahrami S, Vullierme MP, Hammel P, Hentic O, Sauvanet A, Bedossa P, Ruszniewski P and Vilgrain V: Pancreatic endocrine tumours: Tumour blood flow assessed with perfusion $\mathrm{CT}$ reflects angiogenesis and correlates with prognostic factors. Radiology 250: 407-416, 2009.

22. Volm M, Koomägi R and Mattern J: Prognostic value of vascular endothelial growth factor and its receptor Flt-1 in squamous cell lung cancer. Int J Cancer 74: 64-68, 1997.

23. Dong Y, Lei GW, Wang SW, Zheng SW, Ge Y and Wei FC: Diagnostic value of CT perfusion imaging for parotid lesions. Dentomaxillofac Radiol 43: 20130237, 2014.

24. Woo SH, Choi DS, Kim JP, Park JJ, Joo YH, Chung PS, Kim BY, Ko YH, Jeong HS and Kim HJ: Two-phase computed tomography study of warthin tumor of parotid gland: Differentiation from other parotid gland tumors and its pathologic explanation. J Comput Assist Tomogr 37: 518-524, 2013.

25. O'Byrne KJ, Koukourakis MI, Giatromanolaki A, Cox G, Turley H, Steward WP, Gatter K and Harris AL: Vascular endothelial growth factor, platelet-derived endothelial cell growth factor and angiogenesis in non-small-cell lung cancer. Br J Cancer 82: 1427-1432, 2000.

26. Ng QS, Goh V, Milner J, Stratford MR, Folkes LK, Tozer GM, Saunders MI and Hoskin PJ: Effect of nitric oxide synthesis on tumour blood volume and vascular activity: A phase I study. Lancet Oncol 8: 111-118, 2007.

27. Zhang J, Wang R, Lou H, Zou Y and Zhang M: Functional computed tomographic quantification of angiogenesis in rabbit VX2 soft-tissue tumour before and after interventional therapy. J Comput Assist Tomogr 32: 697-705, 2008.

28. Goh V, Halligan S, Daley F, Wellsted DM, Guenther T and Bartram CI: Colorectal tumour vascularity: Quantitative assessment with multidetector CT-do tumour perfusion measurements reflect angiogenesis? Radiology 249: 510-517, 2008.

29. Yao J, Yang ZG, Chen HJ, Chen TW and Huang J: Gastric adenocarcinoma: Can perfusion $\mathrm{CT}$ help to noninvasively evaluate tumour angiogenesis? Abdom Imaging 36: 15-21, 2011.

30. Chen Y, Zhang J, Dai J, Feng X, Lu H and Zhou C: Angiogenesis of renal cell carcinoma: Perfusion CT findings. Abdom Imaging 35: 622-628, 2010.

31. Reinmuth N, Parikh AA, Ahmad SA, Liu W, Stoeltzing O, Fan F, Takeda A, Akagi M and Ellis LM: Biology of angiogenesis in tumours of the gastrointestinal tract. Microsc Res Tech 60: 199-207, 2003. 